\title{
GLUT-1 staining of squamous cell carcinomas of the uterine cervix identifies a novel element of invasion
}

\author{
ARNULF MAYER $^{1}$, MICHAEL HÖCKEL $^{2}$, LARS-CHRISTIAN HORN ${ }^{3}$, \\ HEINZ SCHMIDBERGER ${ }^{1}$ and PETER VAUPEL ${ }^{1}$
}

\author{
${ }^{1}$ Department of Radiooncology and Radiotherapy, University Medical Center, Langenbeckstrasse 1, 55131 Mainz; \\ ${ }^{2}$ Department of Gynecology and Obstetrics, University Medical Center, Liebigstrasse 20a; \\ ${ }^{3}$ Department of Pathology, University Medical Center, Liebigstrasse 26, 04103 Leipzig, Germany
}

Received August 5, 2010; Accepted September 16, 2010

DOI: 10.3892/ijo_00000833

\begin{abstract}
Perturbation of the normal tissue architecture in solid malignant tumors is perceived to be the consequence of actively migrating cancer cells which invade the adjacent normal host tissue. The opposite, invasion of cancer cell clusters by a vascularized stroma, has not been considered. The latter process should, however, be expected to occur since the hypoxic cores of tumor cell aggregates, under the control of HIF-1, are known to secrete cytokines (e.g., bFGF, VEGF) which attract fibroblasts and induce blood vessel formation. In this study, the expression of glucose transporter (GLUT)-1, a major HIF-1 target gene, was examined in 51 squamous cell carcinomas of the uterine cervix by immunohistochemistry to identify the localization of hypoxic tumor cell areas. The relationship of the expression pattern of GLUT-1 with the localization and morphology of the tumor stroma was analyzed. We identified three recurrent histological signs which represent strong evidence in favor of an invasion of solid tumor masses by actively migrating stromal cells. According to our findings, the histological structure of squamous cell carcinomas of the uterine cervix may in part result from the interplay between the inherent tendency of neoplastic epithelial cells to expand in the form of coherent aggregates and the fragmentation of these aggregates by invading, finger- or wedge-like stromal protrusions which carry new blood vessels, driven by gradients of hypoxia-induced pro-angiogenic, pro-migratory and growth-promoting molecules emanating from the hypoxic core.
\end{abstract}

\section{Introduction}

The pathophysiological basis of cancer cell invasiveness is highly complex and heterogeneous. Despite the fact that it is

Correspondence to: Dr Arnulf Mayer, Department of Radiooncology and Radiotherapy, University Medical Center, Johannes Gutenberg University, Langenbeckstrasse 1, 55131 Mainz, Germany E-mail: arnmayer@uni-mainz.de

Key words: invasion, tumor stroma, tumor hypoxia, hypoxiainduced motility, glucose transporter 1, angiogenesis the defining trait of malignant disease, many aspects are still controversial. The phenomenon of single cell invasion has been studied most intensively, but a variety of other mechanisms has also been characterized (1). Since the latter involve complex interactions between tumor and host cells, it is technically much more challenging, if not partially impossible, to reduce these phenomena to in vitro or xenotransplantation analyses. The current emphasis on a mesenchymal-type, single cell spreading mechanism may therefore, at least to a significant extent, be the result of methodological considerations in research practice rather than being a reflection of its relative biological significance. In fact, accumulating evidence indicates that other mechanisms may be more prevalent in vivo, at least in some solid tumor entities (2). An apparent a priori consensus in the current literature, however, is the notion that invasion is generally dominated by actively migrating cancer cells while host cells primarily act as bystanders or fulfill supportive functions. To be more specific, the current view of the function of the tumor stroma in malignant disease is mainly 2-fold: On the one hand, tumor stroma, and in particular the extracellular matrix, which is produced and maintained by stromal fibroblasts, represents an obstacle to cancer invasion. A variety of malignant cells are able to overcome this barrier by the secretion of proteases. On the other hand, subsets of stromal cells play a supportive role in that they provide the cellular basis for angiogenesis, i.e., the outgrowth of new (capillarylike) blood vessels. Since the process of angiogenesis is typically insufficient for the energy/metabolic demand of the evergrowing tumor cell mass, areas of hypoxia, nutrient deprivation, tissue acidosis and metabolic waste accumulation arise.

Cancer cells react to the stress of hypoxia by activating the hypoxia-inducible factor (HIF)-1, a transcription factor which controls a large number of genes involved in the reestablishment of cellular energy homeostasis. Prominent among the processes initiated by HIF-1 is the secretion of pro-angiogenic molecules (e.g., vascular endothelial growth factor, VEGF). HIF-1 also leads to overexpression of growthpromoting and pro-migratory cytokines, e.g., bFGF (3), PDGF and SDF-1 (4). A further consequence of HIF-1 stabilization is the increased expression of high-capacity membrane-bound glucose transporters, most importantly, glucose transporter (GLUT)-1. Although the extent of expression of these molecules in entire tumor sections is not quantitatively correlated to 

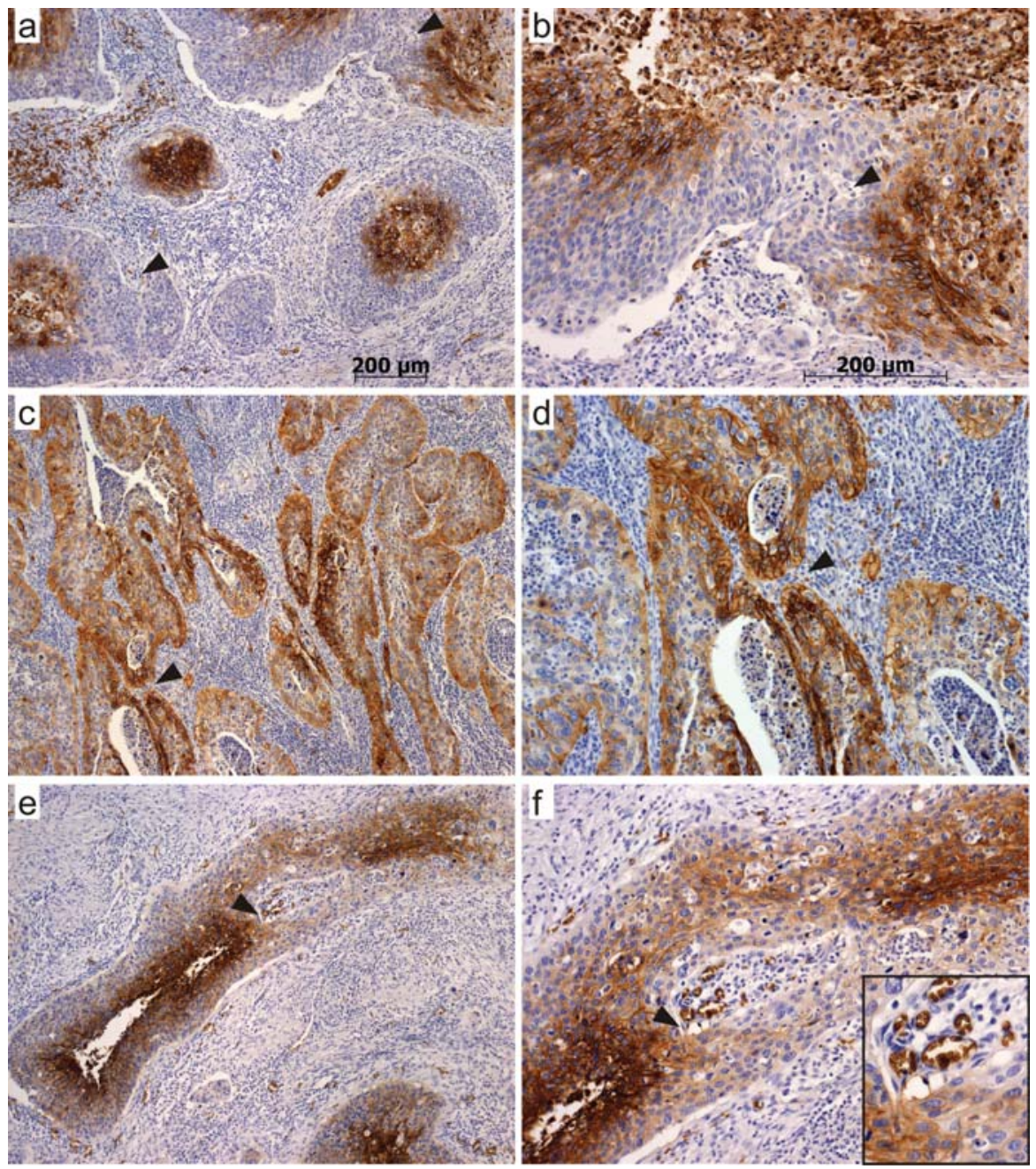

Figure 1. Slices from 3 different squamous cell carcinomas (upper, middle and lower panels) illustrate 2 morphological variations of stromal invasion into cancer cell masses. (a) Both finger-like (lower left) and wedge-like (upper right) protrusions are indicated by arrowheads. (b) A magnified view of the wedge-like structure in (a), which ends in a spike-like tip (arrowhead). Both structures are directed towards the hypoxic core of the tumor cell formations, which can be identified by strong GLUT-1 staining. (c) Multiple finger- and wedge-like protrusions, with prominent examples of the latter being found in the lower left of the image (arrowhead) and magnified in (d). Note the strong expression of GLUT-1 directly adjacent to the stroma, which is highly vascularized, as evident from the presence of multiple GLUT-1-positive RBCs. (e) A finger-like protrusion, which clearly extends from the surrounding stroma to the center of the hypoxic tumor core. Once more, the rich vascularization is evident from GLUT-1 staining of RBCs (f). The inset in (f) shows a magnified view of the spike-like tip of the invading stroma (see Discussion for details). Left panels magnification x10, right panels x20.

the oxygenation status, as shown in a number of publications (5-7), their microregional expression intensity is typically increasing with enlarging distance from blood vessels. Nevertheless, GLUT-1 expression is also regularly found directly adjacent to blood vessels and this phenomenon has been ascribed both to the oxygen-independent activity of HIF-1 and to transient (acute) hypoxia, e.g., due to transitory occlusion of blood vessels (8).

In this study we re-evaluate the typical expression pattern of GLUT-1 in solid tumors drawing on the example of squamous cell carcinomas (SCC) of the uterine cervix. A close examination of histomorphological details, surprisingly, reveals strong evidence for a mechanism of hypoxia-driven invasiveness in which stromal (myo-) fibroblasts may play a previously unrecognized active role.

\section{Patients and methods}

Patients. All patients $(\mathrm{n}=51)$ were part of a prospective clinical study evaluating the significance of intratumoral hypoxia in benign and malignant uterine tumors that took place from 2001 to 2005 at the Department of Gynecology and Obstetrics, University of Leipzig Medical Center. The study was approved by the medical ethics committee of the University of Leipzig Medical Center.

Immunohistochemistry. As described previously (5), histological slides were prepared from the paraffin blocks and dried overnight at $37^{\circ} \mathrm{C}$. The next day, specimens were dewaxed in two changes of fresh xylene and then rehydrated in a descending alcohol series. Retrieval of antigenic binding sites was performed by heating specimens in sodium citrate, $\mathrm{pH} 6.0$, in a steamer (Braun FS 10, Braun, Kronberg, Germany) for 40 min. Rabbit polyclonal anti-GLUT-1 antibodies (cat. no. A3536, dilution 1:200, Dako, Hamburg, Germany) were applied overnight at $4^{\circ} \mathrm{C}$. Biotinylated goat anti-mouse/antirabbit secondary antibodies were applied for $30 \mathrm{~min}$ at $37^{\circ} \mathrm{C}$ followed by the streptavidin/biotin/horseradish peroxidase reagent in accordance with the manufacturer's instructions (Duet kit, Dako). Diaminobenzidine served as the peroxidase 

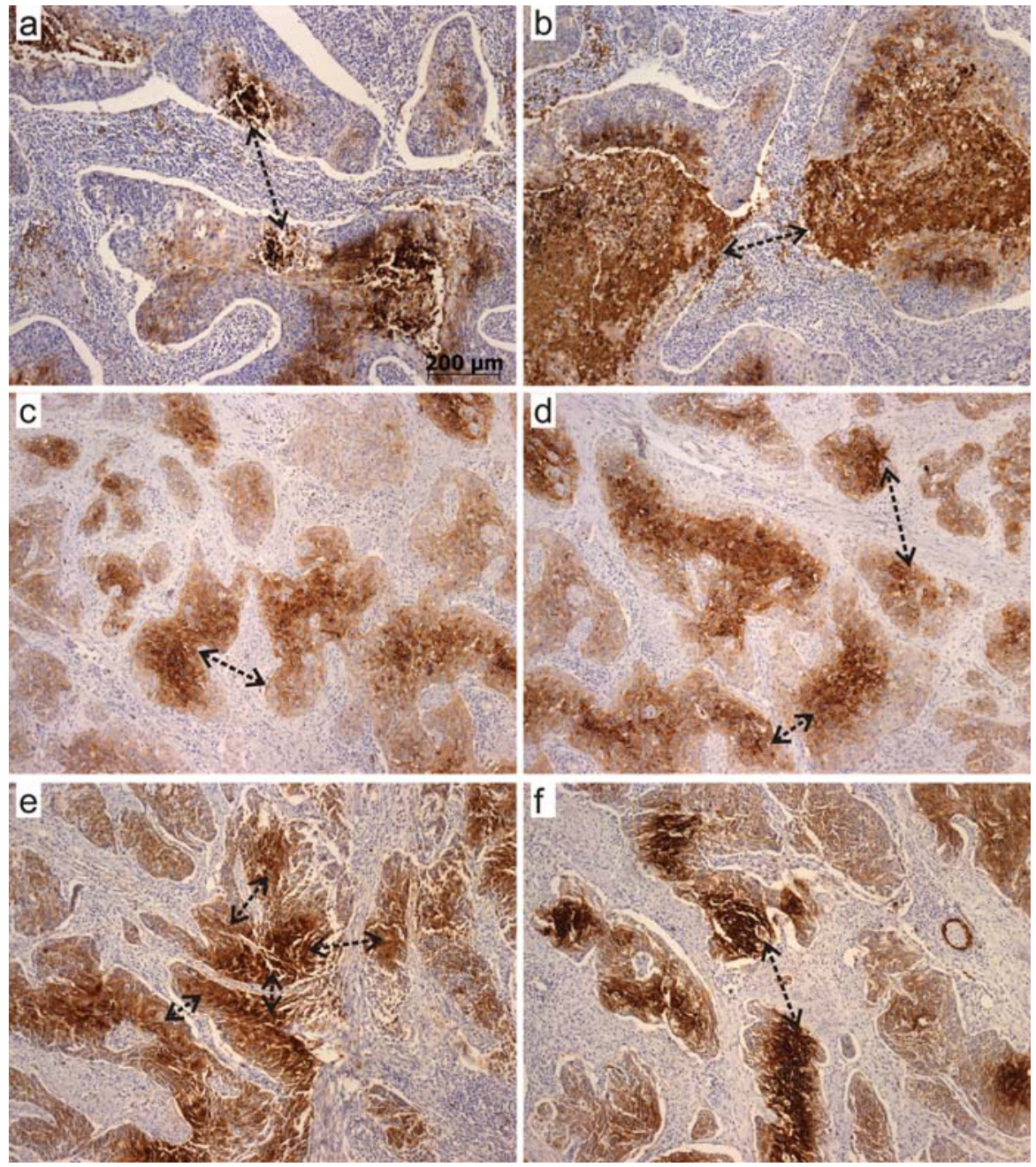

Figure 2. Examples from 3 different squamous cell carcinomas (upper, middle and lower panels) illustrate the 'continental drift' phenomenon, which indicates that tumor cell masses have been separated passively by an actively migrating stroma. Double arrows connected by broken lines denote areas which formerly belonged to one unit. All panels, magnification x10.

substrate. Slides were counterstained with Mayer's hematoxylin, dehydrated in an ascending alcohol series, and covered with a coverslip using Eukitt mounting medium (Riedel-de Haen, Seelze, Germany). Digital images of the specimens were acquired using a Zeiss AxioImager (Zeiss, Oberkochen, Germany) microscope equipped with an AxioCam HRc digital CCD camera and AxioVision software running on a standard Windows-based personal computer.

\section{Results}

The shape of the tumor/stroma interface is indicative of tumor mass infiltration by the stroma. In the 2-dimensional plane of the histological section, tumor cell masses typically show rounded shapes, with blunt edges facing the stroma. The configuration of smaller tumor cell aggregates is often approximately circular with a concentrically configured GLUT-1 positive core. The distance of these GLUT-1 positive cells from the stromal interface is variable. A typical example of these characteristics is shown in Fig. 1a. Stromal cells [e.g., (myo-) fibroblasts, endothelial cells, pericytes] break into the circular formations in the direction of those areas which express GLUT-1 most strongly, i.e., areas which are the most hypoxic. Since GLUT-1 also stains red blood cells, it is also evident that these finger- (lower left of Fig. 1a) or wedge-like (upper right of Fig. 1a and Fig. 1b) stromal protrusions are rich in blood vessels. Fig. 1c also shows finger- and wedge-like stromal protrusions, with the latter being especially prominent in the most strongly GLUT-1 expressing area in the lower left of the figure (Fig. 1c and d). Fig. 1e and $\mathrm{f}$ again show a finger-like-protrusion rich in capillary-like blood vessels (as evident from the presence of GLUT-1 positive RBCs) which, remarkably, ends in a cytoplasmic structure in the shape of a spike (inset in Fig. 1f).

The shape of some tumor cell clusters and GLUT-1 positive areas within indicates that they have been separated passively by an actively migrating stroma. As can be seen from Fig. 2, the shape of some tumor cell masses suggests that adjacent formations are the result of a passive separation by an actively migrating stroma. Finger- and wedge-like protrusions, as discussed above, migrate towards the hypoxic core (GLUT-1 positive). When two or more of these protrusions meet, tumor cell masses are separated passively. Arrows in Fig. 2 indicate GLUT-1 positive areas which, judging from their geometrical configuration, were previously part of one area. 

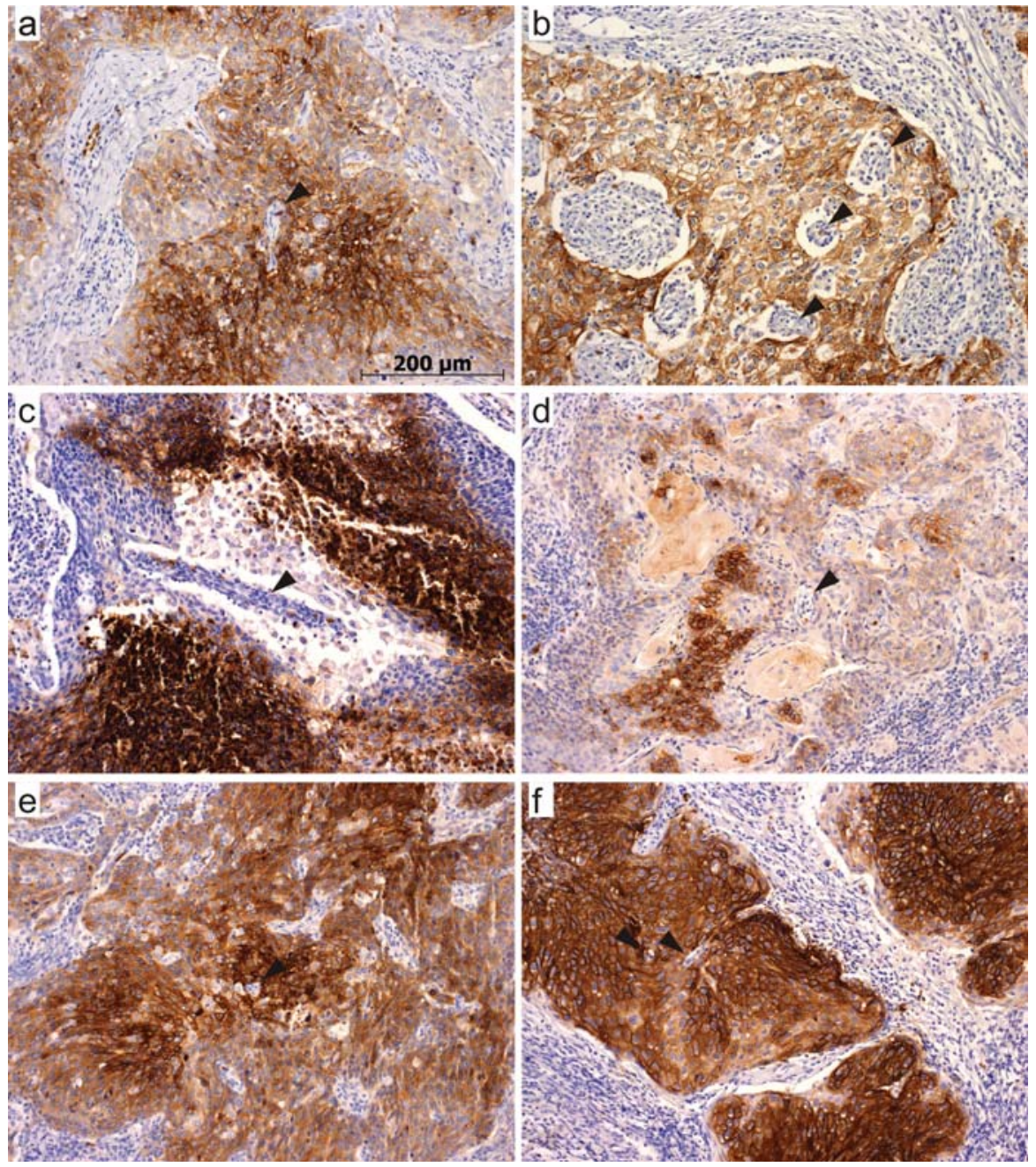

Figure 3. Examples from 6 different squamous cell carcinomas again show multiple finger- and wedge-like protrusions and, additionally, 'entrapped stroma' (arrowheads). The latter can be identified as the most distal part of the cited protrusions in (a, c and f). All panels magnification x20.

Entrapped stroma is found adjacent to the most strongly GLUT-1 expressing tumor cells. Hypoxic tumor cell aggregates are not always finally separated by the stroma. Often, entrapped portions of stroma are found within the tumor cell masses (Fig. 3, arrowheads). They correspond to the most distal ends of the finger- or wedge-like structures as described in Fig. 1, which is evident from the fact that the connection with these protrusions with the surrounding stroma can often be traced back.

Actively invading stroma is widespread in squamous cell carcinomas of the uterine cervix. Forty-three of the 51 (84\%) SCC of the uterine cervix assessed clearly showed one or more of the histological signs [i.e., 1) finger- or wedge-like stromal protrusions, 2) passively separated tumor cell aggregates, 3) entrapped stroma] described above. Only 2 tumors (4\%) did not show this phenotype. In the remaining cases, a definitive decision could not be made due to the lack of sufficient amounts of tumor aggregates in the specimen or because the quality of the histological slide was insufficient (12\%).

\section{Discussion}

In the present study we have shown that results from the immunohistochemical identification of hypoxic areas with antibodies against GLUT-1 may open up new avenues as far as our understanding of mechanisms of hypoxia-driven invasiveness is concerned, as has been demonstrated here by the morphological assessment of the microarchitecture of uterine cervix cancers. We have identified a novel element of cancer invasion, which consists of an actively migrating and infiltrative stroma rich in capillary-like blood vessels. The latter appears to shape the tumor cell masses and, in doing so, decisively influences the histological architecture of the tumor. Importantly, the phenomenon described here is not a peculiarity of some selected tumors or histological subtypes of these squamous cell carcinomas of the uterine cervix. Indeed, the vast majority of the specimens studied here showed one or more of the characteristic histological signs of invasion of vascular stroma into the tumor cell buds. Finger- or wedge-like stromal cell clusters (histological sign 1) which are directed towards the most strongly GLUT-1-expressing areas were reproducibly observed. These structures frequently ended in spike-like extensions of the cytoplasm of the tip cells, which clearly pushed apart the tumor cell aggregates which they invaded. As we have described and illustrated (Fig. 2), two invading protrusions will often meet in center of the hypoxic cell mass. Indicative of this are tumor cell masses which were found to be forced apart in their entirety by the stroma, leaving behind smaller remnants which could still be identified as parts 
of the former unity (histological sign 2) from which they arose, not only by the shape of their boundaries, but also by the outline and localization of GLUT-1 positive areas within (this situation is reminiscent of continental drift where divided land masses can roughly be aligned and additionally contain fossils stemming from identical species). It is difficult to imagine how this morphological structure could have been formed by the active migration of cancer cells, as the currently prevailing hypothesis for the development of the microarchitecture of squamous cell carcinomas postulates. Stromal areas 'entrapped' amidst GLUT-1 positive tumor cells were identified to represent the distal ends of the previously described invasive cell bundles (histological sign 3 ).

In two-dimensional sections, initial foci of cancer cells often have an approximately circular configuration, which in three dimensional space corresponds to planes of rodlike tumor cords cut at right angles to their axis (9). Owing to their continuous proliferation, these aggregates progressively increase in size and, inevitably, develop a hypoxic core, which can be identified by the concentrically shaped GLUT-1 positive interior. Both from published data and from our observations we deduce that an inherent tendency (at least in many subtypes) of squamous cell carcinoma of the uterine cervix is the formation of cohesive tumor cell spheres, a phenomenon well known from and sometimes even spontaneously occurring in in vitro cell culture. These structures may represent the basic configuration which - assuming sufficient supply of nutrients - ex vivo would progress much further (the outer layers of cells would constantly receive sufficient amounts of nutrients to allow continuation of proliferation). However, this growth pattern is regularly disturbed in vivo, but not as a consequence of the cessation of growth in the outer cell layers. Rather, the shift from a spherical growth to the irregular shapes observed in vivo, according to our hypothesis, results from: i) the invasion of the stromal cell compartment into tumor cell aggregates and ii) preferential growth and, arguably, collective migration of cancer cells into directions of least resistance (see below). Cells within the hypoxic (GLUT-1 positive) core of the initial tumor aggregates, by means of activation of HIF-1, up-regulate a plethora of genes, among which GLUT-1 merely served as a marker in our study. More importantly, a number of pro-angiogenic, growth-stimulating and pro-migratory factors are produced by this reaction (10). We argue that these factors subsequently diffuse into the stromal compartment surrounding the initial tumor cell nodule or cord, creating concentric 'hypoxifugal' cytokine gradients. It is well known that the aim of the HIF-1-response is the re-establishment of the nutrient supply of cells in which energy homeostasis is disturbed (10). The means of achieving this goal is also firmly established and consists of the formation of new (capillarylike) blood vessels, i.e., angiogenesis. The spatiotemporal organization and (micro-)architectural consequences of this process in vivo, however, are less obvious. In this respect, extrapolations from established experimental models of angiogenesis (e.g., the chick embryo chorioallantoic membrane assay) may lead to artificial concepts. We advocate the view that capillary-like blood vessels and other components of the stroma in vivo cannot be separated and, hence, 'angiogenesis' denotes outgrowth of (new) stroma which carries blood vessels, rather than outgrowth of isolated blood vessels from the stroma.
In a classic publication from 1986, Dvorak hypothesized that tumors are 'wounds that do not heal' (11). While it is well known that (stromal-derived) granulation tissue (the name refers to its rich vascularization) during wound healing infiltrates fibrin clots formed during hemostasis (12), a similar mechanism, as suggested by this study, has never been considered in cancer pathophysiology. Conversely, it may be argued that the phenomena described in our study solely represent angiogenesis and, therefore, are not novel. However, such a reductionist interpretation would not only ignore possible additional functions of invading stromal cells, but would also miss the decisive element of their destructiveness, which is central in our observations. Infiltrating stroma, following the hypoxia-/HIF-1-induced cytokine gradients described above, pushes towards the tumor cell aggregates from all sides. While molecular details are currently unknown, it is obvious from our study that this process eventually leads to a spatially confined penetration of the 'phalanx' of epithelial cancer cells. Once the cohesion of the cancer cell mass has been broken, the vesselcarrying stroma heads towards the hypoxic core.

While the important role of cancer-associated fibroblasts has been described (13), an active role in tumor invasion has only recently been considered. Using co-culture models of carcinoma cells and human fibroblasts, Gaggioli et al (14) have shown that (in their experimental setup) penetration of an extracellular matrix was performed by fibroblasts, while carcinoma cells followed in their tracks using a collective migration pattern. Conversely, cancer cells were unable to penetrate the native matrix without the aid of fibroblasts. Despite the fact that these data are highly important since they stress the invasive phenotype of fibroblasts, which at present is being largely ignored, the model used by Gaggioli et al (14) is nevertheless artificial, since the influence of the vasculature and diffusion gradients are neglected. Interestingly, Walter-Yohrling et al (15) previously demonstrated that tumor cell spheroids are invaded by myofibroblasts and endothelial cells, but unfortunately, these authors did not discuss in depth the profound implications of their findings as far as in vivo invasiveness is concerned. The results of our morphological studies are partially reminiscent of recent findings by Kilarski et al (16), who proposed a new model of angiogenesis (looping angiogenesis). These authors showed that in the initial phase of neovascularization after wounding, preexisting blood vessels are translocated and expanded as an integral part of 'invading' granulation tissue, in which (myo-) fibroblasts generate tensional force. This model exactly describes the structures shown in Fig. 1, and in particular the one depicted in Fig. 1f. Judging from the configuration of this finger-like protrusion, the whole structure is pulled into the direction of the hypoxic core of a tumor cell aggregate. The connection with the surrounding stroma is clearly visible, as is its abundant vascularization.

It is important to recall that angiogenesis is known to be highly active even in pre-malignant stages of the development of a variety of cancers, particularly in squamous cell carcinomas of the uterine cervix. The question arises, therefore, as to whether even the earliest stage of invasiveness, the penetration of the basal membrane of the normal epithelium, occurs partially as an accidental byproduct of the hypervascularization observed in these lesions. Although the mechanisms of active 
basal membrane degradation by cancer cells as described in textbooks seem to be firmly established, important details may have evaded broader attention (17).

Our hypothesis does not rule out any conventional mechanism of cancer cell invasiveness, be it in the form of single cells or collectives of cells. Since it is increasingly recognized that single cell spreading and epithelial-to-mesenchymal transitions are not prerequisites for (epithelial) cancer invasion (18), there is currently a shift in attention towards collective migration as being of key importance in these entities (19). However, in the light of our findings, it is necessary to point out that: i) collective migration alone does not constitute invasiveness, as evident, e.g., from the aforementioned data by Gaggioli et al (14), ii) experimental studies which investigated this mechanism used cell types which typically also show single cell spreading and extracellular matrix degradation as models [e.g., HT-1080 fibrosarcoma (20)], while iii) in situ zymographic studies of squamous cell carcinomas of the uterine cervix have identified proteolytic activity exclusively in the stromal compartment (21). It is conceivable that an actively migrating and invasive stroma, as described in the present study, may remodel the collagen structure of the extracellular matrix in a way that renders it permissive for the invasion by cancer cell collectives. Recent studies by Provenzano et al elucidated the strong impact of collagen alignment on the motility of cancer cells $(22,23)$.

Höckel et al have recently proposed a concept according to which post-therapeutic recurrences of cancer occur predominantly in the embryonic compartment from which the tumor arose (24). These authors subsequently published clinical data which showed that the surgical resection of cervical cancers, when adapted to take this theory into consideration, yields excellent results (25). Since our data suggest that invasiveness, at least in the earlier stages of cancer progression, is partially mediated by the stromal compartment, our findings strongly support this model. Indeed, it is tempting to speculate that restriction of cancer growth to tissues derived from a common embryonic anlage is the consequence of a lack of responsiveness of stromal cells to signals from neoplastic cells beyond its borders.

In conclusion, using a simple immunohistochemical assay and morphological analyses, we have identified a novel element of invasiveness, which we believe is of key importance for (cervical) cancer growth. Our findings may be of broad relevance not only for surgical concepts, but also for radiotherapy, since this treatment modality is known to have a strong impact on the connective tissue. Further studies are needed to elucidate the molecular details of our observation and, importantly, whether there is an applicability of this mechanism beyond cervical cancer.

\section{Acknowledgements}

We thank Dr Debra Kelleher for her thorough editorial assistance. This study has been supported by grant no. 106758 from the Deutsche Krebshilfe.

\section{References}

1. Friedl $\mathrm{P}$ and Wolf $\mathrm{K}$ : Tumour-cell invasion and migration: diversity and escape mechanisms. Nat Rev Cancer 3: 362-374, 2003.
2. Friedl P and Gilmour D: Collective cell migration in morphogenesis, regeneration and cancer. Nat Rev Mol Cell Biol 10: 445-457, 2009.

3. Calvani M, Rapisarda A, Uranchimeg B, Shoemaker RH and Melillo G: Hypoxic induction of an HIF-1alpha-dependent bFGF autocrine loop drives angiogenesis in human endothelial cells. Blood 107: 2705-2712, 2006.

4. Rankin EB and Giaccia AJ: The role of hypoxia-inducible factors in tumorigenesis. Cell Death Differ 15: 678-685, 2008.

5. Mayer A, Höckel M, Wree A and Vaupel P: Microregional expression of glucose transporter-1 and oxygenation status: lack of correlation in locally advanced cervical cancers. Clin Cancer Res 11: 2768-2773, 2005.

6. Mayer A, Höckel M, Wree A, Leo C, Horn LC and Vaupel P: Lack of hypoxic response in uterine leiomyomas despite severe tissue hypoxia. Cancer Res 68: 4719-4726, 2008.

7. Jankovic B, Aquino-Parsons C, Raleigh JA, et al: Comparison between pimonidazole binding, oxygen electrode measurements, and expression of endogenous hypoxia markers in cancer of the uterine cervix. Cytometry B Clin Cytom 70: 45-55, 2006.

8. Airley R, Loncaster J, Davidson S, et al: Glucose transporter glut-1 expression correlates with tumor hypoxia and predicts metastasis-free survival in advanced carcinoma of the cervix. Clin Cancer Res 7: 928-934, 2001.

9. Thomlinson RH and Gray LH: The histological structure of some human lung cancers and the possible implications for radiotherapy. Br J Cancer 9: 539-549, 1955.

10. Semenza GL: Defining the role of hypoxia-inducible factor 1 in cancer biology and therapeutics. Oncogene 29: 625-634, 2010.

11. Dvorak HF: Tumors: wounds that do not heal. Similarities between tumor stroma generation and wound healing. N Engl J Med 315: 1650-1659, 1986.

12. Singer AJ and Clark RA: Cutaneous wound healing. N Engl J Med 341: 738-746, 1999.

13. Östman A and Augsten M: Cancer-associated fibroblasts and tumor growth-bystanders turning into key players. Curr Opin Genet Dev 19: 67-73, 2009.

14. Gaggioli C, Hooper S, Hidalgo-Carcedo C, et al: Fibroblast-led collective invasion of carcinoma cells with differing roles for RhoGTPases in leading and following cells. Nat Cell Biol 9: 1392-1400, 2007.

15. Walter-Yohrling J, Pratt BM, Ledbetter S and Teicher BA: Myofibroblasts enable invasion of endothelial cells into threedimensional tumor cell clusters: a novel in vitro tumor model. Cancer Chemother Pharmacol 52: 263-269, 2003.

16. Kilarski WW, Samolov B, Petersson L, Kvanta A and Gerwins P: Biomechanical regulation of blood vessel growth during tissue vascularization. Nat Med 15: 657-664, 2009.

17. Weidner N: New paradigm for vessel intravasation by tumor cells. Am J Pathol 160: 1937-1939, 2002.

18. Christiansen JJ and Rajasekaran AK: Reassessing epithelial to mesenchymal transition as a prerequisite for carcinoma invasion and metastasis. Cancer Res 66: 8319-8326, 2006.

19. Friedl $\mathrm{P}$ and Wolf K: Proteolytic interstitial cell migration: a five-step process. Cancer Metastasis Rev 28: 129-135, 2009.

20. Wolf K, Wu YI, Liu Y, et al: Multi-step pericellular proteolysis controls the transition from individual to collective cancer cell invasion. Nat Cell Biol 9: 893-904, 2007.

21. Sier CF, Zuidwijk K, Zijlmans HJ, et al: EMMPRIN-induced MMP-2 activation cascade in human cervical squamous cell carcinoma. Int J Cancer 118: 2991-2998, 2006.

22. Provenzano PP, Eliceiri KW, Campbell JM, Inman DR, White JG and Keely PJ: Collagen reorganization at the tumor-stromal interface facilitates local invasion. BMC Med 4: 38, 2006.

23. Provenzano PP, Inman DR, Eliceiri KW, Trier SM and Keely PJ: Contact guidance mediated three-dimensional cell migration is regulated by Rho/ROCK-dependent matrix reorganization. Biophys J 95: 5374-5384, 2008.

24. Höckel M and Dornhöfer N: The hydra phenomenon of cancer: why tumors recur locally after microscopically complete resection. Cancer Res 65: 2997-3002, 2005.

25. Höckel M, Horn LC, Manthey N, et al: Resection of the embryologically defined uterovaginal (Mullerian) compartment and pelvic control in patients with cervical cancer: a prospective analysis. Lancet Oncol 10: 683-692, 2009. 